\title{
Relaxation model for dynamic plastic deformation of materials
}

\author{
Yuri Petrov ${ }^{1,2}$, Elijah Borodin ${ }^{1,3, a}$, Ezio Cadoni ${ }^{4}$, and Nina Selyutina ${ }^{2}$ \\ ${ }^{1}$ IPME RAS, Extreme States Dynamics Department, V.O., 199178 Bolshoj pr., 61 St. Petersburg, Russia \\ 2 Saint Petersburg State University, 7/9, 199034, Universitetskaya nab., St. Petersburg, Russia \\ ${ }^{3}$ Chelyabinsk State University, 454001 Br. Kashirinykh, 129, Russia \\ ${ }^{4}$ University of Applied Sciences of Southern Switzerland, DynaMatLab, 6952 Canobbio, Switzerland
}

\begin{abstract}
Mechanical model for plastic relaxation of stresses in metals has been proposed. Our approach based on a characteristic plastic relaxation time, which is assumed as a material parameter. In a number of tasks values of its parameter become similarly high and it leads to dynamic effect appearing even at low strain rates deformation condition. The phenomenon of yield drop is one of these manifestations. We also discuss a physically based assumption (in the frames of dislocation theory) for analytical form of relaxation time. We find that there are two different characteristic relaxation time parameters, which is playing a main role for different stages of plastic deformation in low defect crystals.
\end{abstract}

\section{Introduction}

Several cases of unexpected mechanical behavior of metals in deformation can readily be explained by taking their dynamic characteristics into account. One such wellknown paradox is the yield drop [1-3] and sharp yield point phenomenon in metal whiskers [4] when, in the case of quasi-static loading rates $10^{-5}-10^{-2} \mathrm{~s}^{-1}$, the stresses attained in the material are dozens times greater than the value of its quasi-static yield point [4].

In pure metals this effect can be explained in terms of the material "dislocation starvation" [5], when the amount of mobile defects is insufficient for ensuring the required plastic strain rate and the elastic stresses continue to grow. From the mechanical standpoint, this phenomenon expresses the fact that the material already experiences dynamic deformations for such "quasi-static" strain rates. Namely, for a material with such properties, these strain rates are already sufficient for exciting the dynamic deformation mode, which cannot be described on the basis of quasi-static concepts that there are critical flow stresses in the material. To describe the dynamic characteristics of the material, it is necessary to explicitly take into account the fact that the material cannot unboundedly experience plastic relaxations of the arising stresses and a certain time is required for the development of relaxation process inside the material.

This can be attained by introducing an additional dynamic characteristic of the material itself, i.e. the characteristic plastic relaxation time parameter. Obviously, this parameter should be independent of the deformation process characteristics but should express the mechanical properties of the material itself in a wide range of loading rates.

\footnotetext{
${ }^{a}$ Corresponding author: elbor7@gmail.com
}

\section{Models for description of plastic flow of metals}

The elasto-viscoplastic deformation of a solid can be described in the framework of the Voigt model or the Maxwell model of a very viscous fluid [6]. In the Maxwell model, the equation for change in the shear stress $\sigma_{\tau}$ can be written as

$$
\frac{d \sigma_{\tau}}{d t}=2 G \dot{\varepsilon}-\frac{\sigma_{e f f}}{\tau} H\left(\sigma_{e f f}\right)
$$

where $\dot{\varepsilon}$ is the strain rate; $G$ is the shear modulus; $\tau$ is the stress relaxation time such that, under deformation, the material behaves elastically at significantly shorter times and visco-plastically at significantly longer times; $\sigma_{e f f}=$ $\sigma_{\tau}-y_{b}$ is the effective stress such that, at its positive value, the relaxation of shear stresses becomes possible; $y_{b}$ is the barrier stress corresponding to the given mechanism of plasticity; and $H(x)$ is the Heaviside function. The integration of Eq. (1) at a constant strain rate $\dot{\varepsilon}=$ const and the initial condition $\sigma_{\tau}(0)=\sigma_{y}^{0}$ gives the following dependence of the stress on the time:

$$
\sigma_{\tau}(t)=\sigma_{y}^{0}+2 G \tau \dot{\varepsilon}(1-\exp (-t / \tau)) .
$$

For $\dot{\varepsilon}=0$, we have $\sigma_{\tau}(t)=\sigma_{y}^{0}$. At a fixed strain rate, the maximum shear stresses achieved in the material at $t \rightarrow \infty$ can be represented in the form

$$
\sigma_{\tau}^{\max }=\sigma_{y}^{0}+2 G \tau \dot{\varepsilon} .
$$

These stresses determine the dynamic yield strength of the material and consist of two parts: the barrier stress $\sigma_{y}^{0}$ and the "dynamic addition" $2 G \tau \dot{\varepsilon}$, which depends linearly on the strain rate, the characteristic relaxation time, and the elastic constants of the metal.

In terms of the aforementioned models of an elastoviscoplastic body, there are no fundamental differences 
between the shear strengths under the quasi-static and dynamic deformation conditions. Everything is determined by the parameters accounting for the internal structure and defect substructure of the material. The transition between the static and dynamic regimes, according to formula (3), occurs at a strain rate $\dot{\varepsilon}_{t r} \sim \sigma_{y}^{0} / 2 G \tau \equiv \sigma_{y}^{0} / \mu$, where the coefficient $\mu=2 G \tau$, which characterizes the dynamic viscosity, depends on the elastic properties and defect substructure of the metal. For different concentrations of defects in the material, the strain rate can take almost any value. As an example, we consider the dislocation glide in which the plastic strain rate is determined by the Orowan equation [7-9]

$$
\dot{\varepsilon}_{p l}=\sum_{\beta} \rho_{D}^{\beta} b V_{D}^{\beta}
$$

where $\rho_{D}^{\beta}$ is the scalar dislocation density, which is numerically equal to the length of dislocation lines per unit volume; $b$ is the Burgers vector; and is the $V_{D}^{\beta}$ magnitude of the dislocation velocity in the slip system $\beta$. According to this equation, for a specified plastic strain rate, it is necessary to use a particular dislocation density $\rho_{D}^{(c r)}=\dot{\varepsilon}_{p l} / b V_{D}^{\beta}$ for the effective relaxation of shear stresses generated in the material (for the fulfillment of the condition $\dot{\varepsilon}_{p l} \sim \dot{\varepsilon}$ ). The dislocation velocity cannot exceed the transverse velocity of sound in the metal [10]. If the density of dislocations is less than $\rho_{D}^{(c r)}$, their amount is insufficient to ensure the required plastic strain rate, which will lead to a sharp increase in the yield strength of the metal and to the manifestation of its dynamic features even at low strain rates. The phenomenon of the lack of dislocations is well known in the physics of nano-objects, where it is referred to as the dislocation starvation of the material [11]. As will be shown below, the characteristic time of plastic relaxation is inversely proportional to the dislocation velocity and increases without limit when $\rho_{D} \rightarrow 0$. Therefore, even at a low barrier resistance $y_{b}$ under quasi-static deformation conditions, the yield strength can increase up to values of the theoretical tensile strength, which is observed in pure single crystal whiskers. The transition strain rate $\dot{\varepsilon}_{t r}$, at which the dynamic effects become significant, in this case, can be arbitrarily small. Such an increase in the yield strength proves to be natural for extremely high strain rates, when normal dislocation densities $\rho_{D} \sim 10^{12} \mathrm{~m}^{-2}$ are no longer sufficient to ensure the required plastic strain rate. In the dislocation starvation relaxation time is determined by the rate of generation of new defects in the crystal lattice [12].

The similar formalism is used in the yield criterion proposed in $[13,14]$, where it was also shown that the explicit introduction of the characteristic relaxation time makes it possible to determine the yield strength for an arbitrary law of loading of a material and to consider, within a unified model, both the fast and slow loading. For a Voigt body, the strain dependence of the stress is described by the formula

$$
\sigma=2 G \varepsilon+\mu \dot{\varepsilon},
$$

which can also be rewritten as $\dot{\varepsilon}+2 G \varepsilon / \mu=\sigma / \mu$. It is assumed that, apart from the elastic strains, under dynamic conditions there is a viscous addition proportional to the strain rate. The integration of expression (4) with respect to time by multiplying both sides by $2 G \exp (-t / \tau)$ gives

$$
2 G \varepsilon=\frac{1}{\tau} \int_{0}^{t} \sigma(s) \exp \left(-\frac{t+s}{\tau}\right) d s,
$$

where we introduced the characteristic time $\tau=\mu / 2 G$.

The left hand side of this equation represents the elastic stress that we would have in the quasi-statics by setting $\dot{\varepsilon}=0$ in formula (4). Equation (5) can be interpreted in the framework of the concept of fading memory [15]: the load acting earlier at instants of time $s \ll t$ made a significantly smaller contribution to the current state of the defect structure as compared to the recently applied load. The current stress should be replaced by its "relaxed" value. Moreover, it should be noted that the stresses acting in the material at previous instants of time are included in the equation with smaller and smaller weighting coefficients. In the general case, this leads to the inequality in the integral form

$$
\int_{0}^{t} \sigma(s) K(t-s) d s \leq \sigma_{y}^{0}
$$

where the kernel of the integral operator $K(t)$ is the fading memory function. By comparing expressions (6) and (7), we find that the Voigt model corresponds to the exponential memory decay:

$$
K(t)=\frac{1}{\tau} \exp \left(-\frac{t}{\tau}\right)
$$

where, as in expression (6), the $\tau=\mu / 2 G$ is the characteristic time. As a simpler approximation of the exponential law (7), we can propose the step decay function

$$
K(t)=\left\{\begin{array}{l}
\frac{1}{\tau}, 0 \leq t \leq \tau \\
0, t>\tau
\end{array}\right.
$$

Eq. (7) then gives the integral yield criterion for a metal in the form $[7,8]$

$$
\frac{1}{\tau} \int_{t-\tau}^{t}\left(\frac{\sigma(s)}{\sigma_{y}^{0}}\right)^{\alpha} d s \leq 1
$$

where $\sigma(s)$ is the function specifying the time dependence of the applied stresses, which are assumed to be zero at $t<0$ [14]; $\tau$ is the characteristic time of stress relaxation ("incubation time") $[13,14] ; \sigma_{y}^{0}$ is the yield strength under quasi-static loading and $\alpha$ is the dimensionless parameter describing the sensitivity of the material to the level of stresses, usually $\alpha \geq 1$ [14].

We shall assume that the deformation in the specimen increases linearly as $\varepsilon(t)=\dot{\varepsilon} t H(t)$, where $H(t)$ is the Heaviside function. We introduce the dimensionless relaxation function $0<\gamma(t) \leq 1$ by the condition:

$$
\gamma(t)=\left\{\begin{array}{lr}
1 & , \frac{1}{\tau} \int_{t-\tau}^{t}\left[\frac{\Sigma(s)}{\sigma_{y}^{0}}\right]^{\alpha} d s \leq 1 \\
\left\{\frac{1}{\tau} \int_{t-\tau}^{t}\left[\frac{\Sigma(s)}{\sigma_{y}^{0}}\right]^{\alpha} d s\right\}^{-1 / \alpha}, \frac{1}{\tau} \int_{t-\tau}^{t}\left[\frac{\Sigma(s)}{\sigma_{y}^{0}}\right]^{\alpha} d s \leq 1
\end{array}\right.
$$


Here $\Sigma(t)=2 G \dot{\varepsilon} t H(t)$ is the function coinciding with the stress in the specimen at the stage of elastic deformation, i.e., before the time $t_{*}$ of appearance of the macroscopic plastic flow; this time is calculated by criterion (9). The actual stresses in the deformed specimen for $t \geq t_{*}$ is determined by the relation

$$
\sigma(t)=2 G g(t) \varepsilon(t)
$$

where $g(t)=G \gamma^{1-\beta}(t)$ and $\beta$ is a scalar parameter $(0 \leq \beta \leq 1)$ which controls the strengthening degree. (The case $\beta=0$ corresponds to the absence of strengthening.) In the case where the stress relaxation is caused by the simultaneous action of different mechanisms of plasticity, it is advisable to introduce into the model several characteristic relaxation times [16] or, in a more general case, to specify a function describing the contribution from processes with different characteristic times.

\section{Numerical simulation and discussion}

We shall investigate well-known yield drop phenomenon in metal with low dislocation density. A large amount of tensile experiments demonstrate that even in the case of quasi-static loading rates $10^{-4}-10^{-2} \mathrm{~s}^{-1}$, the stresses attained in the material can be dozens times greater than the value of its quasi-static yield point [1-5]. When the amount of mobile defects is insufficient for ensuring the required plastic strain rate and the elastic stresses continue to grow. From the mechanical standpoint, this phenomenon expresses the fact that the material already experiences dynamic deformations for such "quasistatic" strain rates. Namely, for a material with such properties, these strain rates are already sufficient for exciting the dynamic deformation mode, which can not be described on the basis of quasistatic concepts that there are critical flow stresses in the material. To describe the dynamic characteristics of the material, it is necessary to explicitly take into account the fact that the material cannot unboundedly experience plastic relaxations of the arising stresses and a certain time is required for the development of relaxation process inside the material. This can be attained by introducing an additional dynamic characteristic of the material itself, i.e., the characteristic plastic relaxation time parameter. Obviously, this parameter should be independent of the deformation process characteristics but should express the mechanical properties of the material itself in a wide range of loading rates.

\subsection{Numerical simulations of yield drop phenomenon}

Figure 1 shows an example of the calculation of the stressstrain curve for a linear-in-time increase of the strain according to formulas (9)-(11) for a hypothetical material with strain hardening, which roughly corresponds to a soft steel $\left(G=100 \mathrm{GPa}, \sigma_{y}^{0}=200 \mathrm{MPa}, \beta=0.18, \dot{\varepsilon}=\right.$ $\left.8.5 \times 10^{-3} \mathrm{~s}^{-1}\right)$. Fitting relaxation time is $0.4 \mathrm{~s}$ that two orders of magnitude smaller then for whiskers samples of metals [18] and many orders of magnitude more than in usual metal samples.

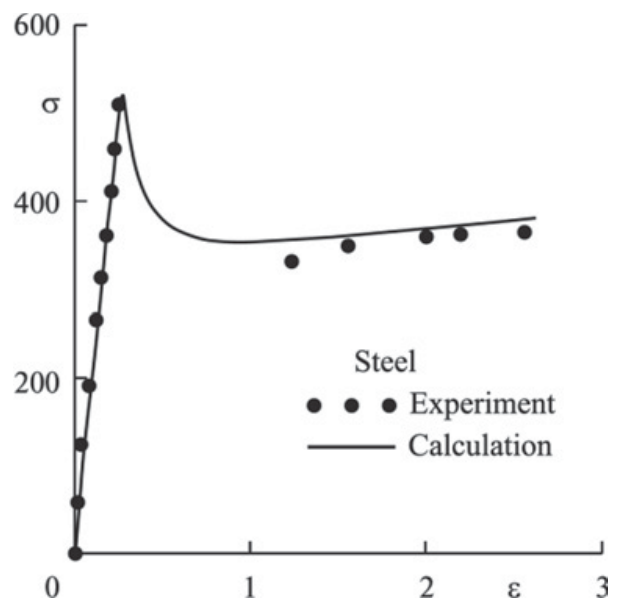

Figure 1. Sharp yield point effect with the subsequent strain hardening in the soft steel at a strain rate of $8.5 \times 10^{-3} \mathrm{~s}^{-1}$. The calculation is performed according to model (9)-(11). Dots are the experimental data taken from [1].

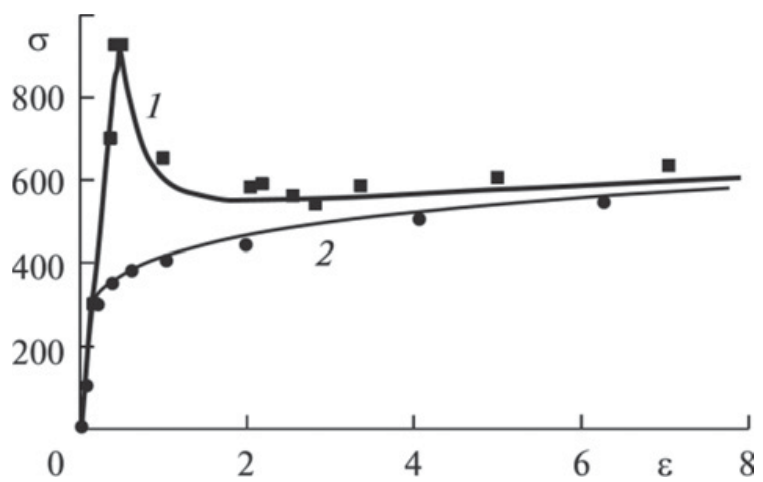

Figure 2. Steel specimens ( $\left.G=78 \mathrm{GPa}, \sigma_{y}^{0}=310 \mathrm{MPa}, \alpha=1\right)$. The points correspond to the experimental data given in [17].

Figure 2 illustrates the simulation of the sharp yield point effect with the subsequent deformation strengthening according to the model (9)-(11) in the case of deformation of steel specimens $\left(G=78 \mathrm{GPa}, \sigma_{y}^{0}=310 \mathrm{MPa}, \alpha=1\right)$. The points correspond to the experimental data given in [17]. In this material, the sharp yield point effect is already not observed at quasi-static strain rates 5 . $10^{-2} \mathrm{~s}^{-1}$ (curve 2), but is clearly observed in the case of dynamic deformation at the rates $10^{3} \mathrm{~s}^{-1}$ (curve 1). The points correspond to the recently obtained experimental data [17]. It is remarkable that in this case the obtained value of relaxation time scale is already equal only to 14 microseconds that are still too many.

Figures 3 and 4 show dependences stress of iron on deformation degree under strain rate in the order $10^{-3}$ based on (9)-(11). The constant characteristic time in Fig. 3 is increased upon condition decrease of the dislocations density (curve $1-10 \mathrm{~cm}^{-2}$, curve $2-10^{3} \mathrm{~cm}^{2}$, curve $3-$ $10^{5} \mathrm{~cm}^{-2}$, curve $4-10^{7} \mathrm{~cm}^{-2}$ ) and growth of the "yield drop" peak.

As shown in Fig. 4, the behavior of iron under deformations characterized by dependence with static yield stress $145 \mathrm{MPa}$ and less constant characteristic time 0.46 versus parameters in Fig. 3 . 


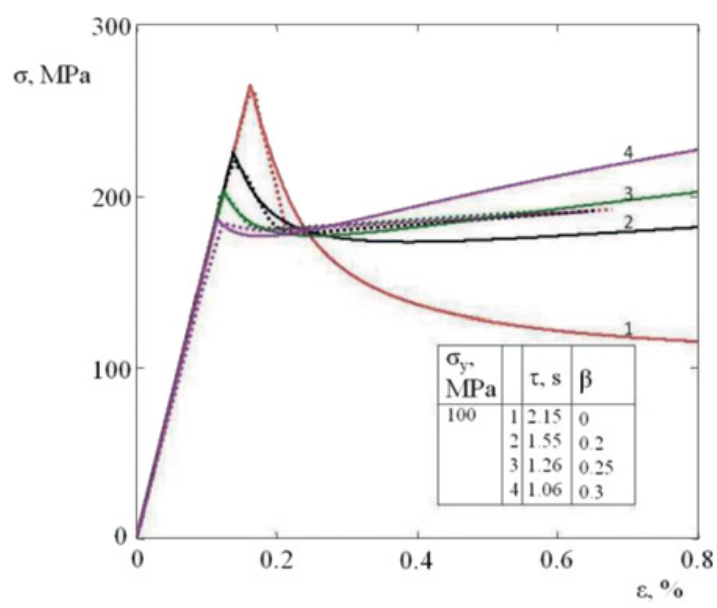

Figure 3. The stress-strain curves for small deformations of iron with the single relaxation time parameter. Initial dislocation densities for curves are (1) $-10 \mathrm{~cm}^{-2},(1)-10^{3} \mathrm{~cm}^{-2}$, (3) $-10^{5} \mathrm{~cm}^{-2},(4)-10^{7} \mathrm{~cm}^{-2}$. Our simulation and data of G.T. Hahn (dots) [5].

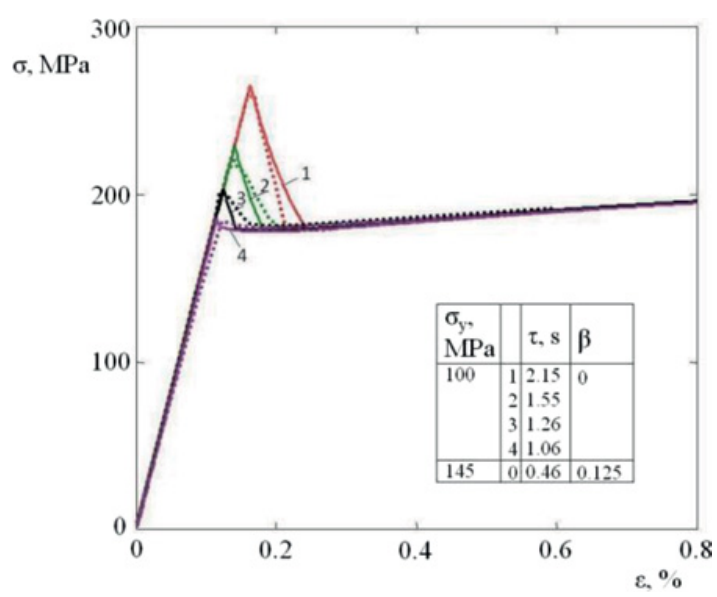

Figure 4. The stress-strain curves for small deformations of iron with several relaxation time parameters. Initial dislocation densities for curves are (1) $-10 \mathrm{~cm}^{-2}$, (2) $-10^{3} \mathrm{~cm}^{-2}$, (3) $-10^{5} \mathrm{~cm}^{-2},(4)-10^{7} \mathrm{~cm}^{-2}$. Our simulation and data of G.T. Hahn (dots) [5].

Assuming that the process deformation of iron consists in phenomena of "yield drop" and further hardening of the material related to different parameters incubation time and yield stress, stress-strain piecewise function is plotted in Fig. 4. The parameter of the hardening $\beta$ is equal to zero in "yield drop" part. The relative transition point between two processes for each curve is calculated on incubation time of "yield drop" part and time beginning plastic deformation of "hardening" part.

As shown above, decrease of the dislocations density result in increase incubation time on "yield drop" part. Thus, the characteristic time of "yield drop" part can be involved with kinetic of defects, introducing hardening parameter $\beta$ in relaxation function $\gamma(t)$ on second part curve in Fig. 3. On account of the macroscopic parameters of the yield stress and incubation time can be characterized stress-strain curve in view of micro-defects.

\subsection{Physical based interpretations for relaxation time parameters}

In the case of a steady-state flow, the expression (2) determines the level of maximal stresses attained in the material, and it can be rewritten as $\sigma_{\tau}^{\max }(3)$. The maximum shear stresses (3) are proportional to the yield point of the material. It follows from (3) that, in the case of small characteristic times and strain rates, we have a constant value of the yield point, which can vary only due to the strain strengthening [19]. One can determined static yield strength $\sigma_{y}^{0}$ by the Taylor and Hall-Petch lows $[16,19]$. On the other hand, at high strain rates or at large characteristic relaxation times, the second term in (3) becomes significant and the dependence of the yield point on the strain rate is rather complicated.

We use Eq. (3) to estimate the characteristic time of plastic relaxation due to the dislocation sliding. Consider the dislocation model of crystal plasticity, which states that the plastic strain is caused by displacements of separate defects of the crystal structure [19]. At times exceeding the acceleration time of dislocations (of the order of $10^{-10_{-}}$ $10^{-9} \mathrm{~s}$ ), we can assume that their motion is steady-state and $\dot{\sigma}=0$. Then, under the condition that the high external stresses are significantly greater than the barrier stresses $\sigma_{\tau} \gg \sigma_{y}$, it follows from (3) that

$$
\sigma_{\tau}=2 G \tau \dot{\varepsilon} .
$$

In [13], it was shown that, by substituting (3) into the Orovan relation (10) and by using the dislocation dynamic equation $[16,18]$, we obtain the following expression for the characteristic stress relaxation time:

$$
\tau_{D}=\frac{B_{f}}{G b^{2} \rho_{D}} \sim \frac{B_{f}}{E_{D}}
$$

where $E_{D}$ is the total elastic energy of dislocation lines per unit volume and $B_{f}=\left(v_{D} / 2\right) \rho b^{2}$ characterizes the rate of scattering of the dislocation kinetic energy [20]; $v_{D} \sim 10^{13} \mathrm{~s}^{-1}$ is the nearly Debye frequency whose inverse value gives the time of scattering of the kinetic energy of mobile dislocations and $\rho$ is the material density. The time (13) is inversely proportional to the dislocation density and can vary in sufficiently broad ranges. At the initial deformation stages, which are important for the sharp yield point effect, the relaxation time is completely determined by the density of dislocations which are present in the material before the deformation. That is true for metal wishkers with extremely low initial dislocation density [18]. For yield drop phenomenon one can see from Fig. 3, Fig. 4 that relations of characteristic time to dislocation density has logarithmic form. Similar relation has the time needs for increasing of dislocation density $[8,9]$.

$$
\tau_{w} \sim \frac{\varepsilon_{D}}{0.2} \frac{\ln \left(\rho_{D}^{c r} / \rho_{D}^{0}\right)}{B V_{D}^{2}},
$$

here $V_{D}$ velocity of dislocations relative to the substance, $\varepsilon_{D} \sim 8 \mathrm{eV} / \mathrm{b}$ is the energy of the dislocations formation per unit length $[9,20]$.

From the standpoint of the above-mentions models of an elastoviscoplastic body, there are no fundamental 
differences between the shear strength in the case of "quasi-static" strain and in the case of "dynamic" strain. Everything is determined by the values of the parameters describing the internal structure and the defect substructure of the material. For various defect concentrations in the material, this strain rate can take practically any values. The defect structure and the mechanical properties of the material vary in the course of plastic deformation.

\section{Conclusion}

Thus, the dynamic deformation mode is determined not by external factors but by the relation between the strain rate and the material ability to relaxation of elastic stresses arising in it. The value of the characteristic plastic relaxation time determines the relaxation properties of the material and is one of its basic dynamic characteristics. This time is independent of the strain rate and is determined at the micro level by the dynamic properties of the basic carriers of plastic deformation and by their amount per unit volume. The coincidence of the values of characteristic time obtained by various approaches confirms the claim that its nature is universal. At relatively small strains, the mechanical properties are determined by a single value of the characteristic time corresponding to the initial dislocation density. For large deformation nature of the characteristic relaxation time can may change. Also at strong plastic deformations, it is necessary to take into account the irreversible changes in the defect structure of the material, which can be described by simple kinetic equations for the dislocation density variations. Macroscopically, this can be expressed as a decrease in the characteristic time of plastic deformation with time. In the case of deformation of pure metals with low dislocation density the characteristic relaxation time turns out to be very large; i.e., it is several orders of magnitude greater than the relaxation times typical of usual specimens of metals with large dislocation densities [14].

The research was supported by the Program of the President of the Russian Federation (Grant No. MD-286.2014.1), by the Russian Foundation for Basic Research (Grants Nos. 14-0131454 and 14-01-00814), by Program No. 25 of Presidium of the Russian Academy of Sciences, by Marie Curie Foundation TAMER No. 610547, and the Ministry of Education and Science of Russian Federation (competitive part of State Task of NIR CSU No. 3.1334.2014/K).

A special acknowledgement goes to the Scientific \& Technological Cooperation Programme Switzerland-Russia for the financial support of the Utilisation of Specific Infrastructure Project between DynaMat Laboratory of the University of Applied Sciences of Southern Switzerland and the St. Petersburg State University.

\section{References}

[1] M.M. Hutchinson, J. Iron Steel Inst. 186 (1957)

[2] S.S. Brenner, J. Appl. Phys. 28, 9 (1957)

[3] B.V. Petukhov, Technical Physics 71, 11 (2001)

[4] R.E. Smallman, R.J. Bishop Metals and Materials: Science, Processes, Applications (ButterworthHeinemann Ltd, 1995)

[5] G.T. Hahn, Acta Met. 10, 17 (1962)

[6] L.D. Landau, E.M. Lifshitz, Course of theoretical physics, Vol. 7: Theory of elasticity (Nauka, Moscow, 2003; Butterworth-Heinemann, Oxford, 2005)

[7] E.N. Borodin, A.E. Mayer, Phys. Solid State. 54, 4 (2012)

[8] V.S. Krasnikov, A.E. Mayer, A.P. Yalovets, Int. J. Plast. 27 (2011)

[9] A.E. Mayer, K.V. Khishchenko, P.R. Levashov, P.N. Mayer, J. Appl. Phys. 113 (2013)

[10] Shock waves and high-strain-rate phenomena in metals, Ed. by M. A. Meyers and L. E. Murr (Plenum, New York, 1981; Metallurgiya, Moscow, 1984)

[11] J.R. Greer, J.Th.M. De Hosson, Prog. Mater. Sci. 56 (2011)

[12] R.W. Armstrong, F.J. Zerilli, J. Phys. D: Appl. Phys. 43 (2010)

[13] A.A. Gruzdkov, Yu.V. Petrov, V.I. Smirnov, Phys. Solid State 44, 11 (2002)

[14] A.A. Gruzdkov, E.V. Sitnikova, N.F. Morozov, Y.V. Petrov, Math. Mech. Solids 14, 72 (2009)

[15] Yu.N. Robotnov, Elements of hereditary solid mechanics (Nauka, Moscow, 1977; Mir, Moscow, 1980)

[16] E.N. Borodin, A.E. Mayer, Yu.V. Petrov, A.A. Gruzdkov, Physics of the Solid State, 56, 12 (2014)

[17] E. Cadoni, F. D'Aiuto, and C. Albertini, “ Dynamic Behavior of Advanced High Strength Steel Used in the Automobile Structures," DYMAT 1, 135-141 (2009)

[18] Yu.V. Petrov, E.N. Borodin, Physics of the Solid State, 57, 2 (2015)

[19] M.A. Meyers, K.K. Chawla, Mechanical behavior of materials (Cambridge Univ. Press, New York, 2009)

[20] A.E. Dudorov, A.E. Mayer, Vestnik Chelyabinsk Gos. Univ. 39, 12 (2011) 